\title{
A PRIORI ESTIMATES IN WEIGHTED SPACES FOR SOLUTIONS OF THE POISSON AND HEAT EQUATIONS
}

Abstract. We prove a priori estimates for solutions of the Poisson and heat equations in weighted spaces of Kondrat'ev type. The weight is a power of the distance from a distinguished axis.

1. Introduction. In this paper we obtain a priori estimates for solutions of the Poisson and heat equations in weighted spaces of Kondrat'ev type in $\mathbb{R}^{3}$. Here the weight is some power of the distance from a distinguished axis in $\mathbb{R}^{3}$ and the power depends on the order of the derivative of the function. We want to stress that the weights we are concerned with are not $\mathrm{A}_{2}$ weights (i.e. Muckenhoupt's weights, see [Mc72]). This is the main difficulty in deriving a priori estimates.

Solutions which belong to weighted spaces of Kondrat'ev type can be found in e.g. [Na94], [Ko97], where the authors examine boundary value problems in dihedral domains. In our investigations we replace the dihedral domain by $\mathbb{R}^{3}$ with the $z$-axis removed. We deduce a priori estimates for solutions of the Poisson (resp. heat) equation in the space $H_{\mu}^{2}\left(\mathbb{R}^{3}\right.$ ) (resp. $\left.H_{\mu}^{2,1}\left(\mathbb{R}^{3} \times \mathbb{R}_{+}\right)\right)$in three steps. First, utilizing Kondrat'ev's method [Ko67] we examine the Poisson equation in $\mathbb{R}^{2}$ and we show that, if $\mu$ is noninteger, then for each $f \in L_{2, \mu}\left(\mathbb{R}^{2}\right)$ there exists a unique solution $u \in H_{\mu}^{2}\left(\mathbb{R}^{2}\right)$ of $\Delta u=f$. Next we obtain estimates in weighted spaces for solutions of a related two-dimensional elliptic problem with a parameter. This is the main step of the proof. Finally, we obtain a priori estimates in the weighted spaces $H_{\mu}^{2}\left(\mathbb{R}^{3}\right)$ and $H_{\mu}^{2,1}\left(\mathbb{R}^{3} \times \mathbb{R}_{+}\right)$.

2000 Mathematics Subject Classification: 35J05, 35B45.

Key words and phrases: weighted space, a priori estimate, Poisson equation, heat equation.

The research is partially supported by MNiSW grant no 1 P03A 02130. 
Proving a priori estimates is the crucial step in examining the uniqueness of solutions of the Poisson and heat equations. The problem of existence of solutions will be explored in another paper. We mention that the weighted spaces which we consider in this paper also appear in [Za04], where the author studies the Navier-Stokes equations. The a priori estimates given here can be a useful tool in examining some problems relating to the N-S equations.

Our main results are the following:

Theorem 1. Assume that $\mu \in(-1,0)$ and $n=-1,0,1,2, \ldots$ Then there exists a constant $c=c(\mu, n)$ such that for each $u \in H_{\mu-n}^{2}\left(\mathbb{R}^{3}\right)$,

$$
\|u\|_{H_{\mu-n}^{2}\left(\mathbb{R}^{3}\right)} \leq c\|\Delta u\|_{L_{2, \mu-n}\left(\mathbb{R}^{3}\right)} .
$$

Theorem 2. Assume that $\mu \in(-1,0), n=-1,0,1,2 \ldots$ Then there exists a constant $c=c(\mu, n)$ such that for each $u \in H_{\circ}^{2,1}\left(\mathbb{R}^{3} \times \mathbb{R}_{+}\right)$,

$$
\|u\|_{H_{\mu}^{2,1}\left(\mathbb{R}^{3} \times \mathbb{R}_{+}\right)} \leq c\left\|u_{t}-\Delta u\right\|_{L_{2, \mu-n}\left(\mathbb{R}^{3} \times \mathbb{R}_{+}\right)} .
$$

The definitions of the weighted function spaces appearing above are given in the next section.

2. Notation. We denote by $r=r(x)$ the distance of $x \in \mathbb{R}^{n}$ from the set $\left\{x: x_{1}=x_{2}=0\right\}$. For $n=2,3$ we define $\dot{\mathbb{R}}^{n}=\mathbb{R}^{n} \backslash\left\{x \in \mathbb{R}^{n}: r=0\right\}$, and $\mathcal{D}\left(\dot{\mathbb{R}}^{n}\right)$ is the set of smooth functions with compact support in $\dot{\mathbb{R}}^{n}$.

We recall the standard notation of function spaces: if $U \subseteq \mathbb{R}^{n}$, then $L_{2, \mu}(U):=\left\{u: u \cdot r^{\mu} \in L^{2}(U)\right\}$ with the norm $\|u\|_{L_{2, \mu}(U)}:=\left\|u \cdot r^{\mu}\right\|_{L^{2}(U)}$. The space $H_{\mu}^{m}\left(\mathbb{R}^{n}\right)$ is defined as the closure of the set $\mathcal{D}\left(\dot{\mathbb{R}}^{n}\right)$ with respect to the norm

$$
\|u\|_{H_{\mu}^{m}(U)}:=\left\{\sum_{|\alpha| \leq m}\left\|D^{\alpha} u\right\|_{L_{2, \mu-m+|\alpha|}(U)}^{2}\right\}^{1 / 2} .
$$

Finally, if $\mathbb{R}_{+}:=\{t: t>0\}$, then the space $H_{0}^{2,1}\left(\mathbb{R}^{n} \times \mathbb{R}_{+}\right)$is defined as the closure of the set $\mathcal{D}\left(\dot{\mathbb{R}}^{3} \times \mathbb{R}_{+}\right)=\{u: u$ smooth with supp $u$ compact in $\left.\dot{\mathbb{R}}^{3} \times \mathbb{R}_{+}\right\}$with respect to the norm

$$
\|u\|_{H_{\mu}^{2,1}\left(\mathbb{R}^{n} \times \mathbb{R}_{+}\right)}=\left\{\sum_{|\alpha|+2 s \leq 2}\left\|D_{t}^{s} D_{x}^{\alpha} u\right\|_{L_{2, \mu}\left(\mathbb{R}^{n} \times \mathbb{R}_{+}\right)}^{2}\right\}^{1 / 2} .
$$

Let

$$
Q_{1}:=\mathbb{R} \quad \text { and } \quad Q_{2}:=\mathbb{R}^{2}
$$

and let $s=s(q)$ be defined on $Q_{1}$ or $Q_{2}$ by

$$
s(q)= \begin{cases}q^{2} & \text { for } q \in Q_{1}, \\ q_{1}^{2}+i q_{2} & \text { for } q=\left(q_{1}, q_{2}\right) \in Q_{2} .\end{cases}
$$


3. Problem in $\mathbb{R}^{2}$. In this section we consider the Poisson equation in $\mathbb{R}^{2}$ using Kondrat'ev's method (see [Ko67]). We prove that if $\mu$ is noninteger, then for each $f \in L_{2, \mu}\left(\mathbb{R}^{2}\right)$ there exists a unique $u \in H_{\mu}^{2}\left(\mathbb{R}^{2}\right)$ which satisfies $\Delta u=f$. In order to get this result we have to examine the following problem with a parameter:

$$
\left\{\begin{array}{l}
-v_{\varphi \varphi}+\lambda^{2} v=g \quad \text { in }(0,2 \pi), \\
v(0)=v(2 \pi) \\
v_{\varphi}(0)=v_{\varphi}(2 \pi)
\end{array}\right.
$$

where $v_{\varphi}$ and $v_{\varphi \varphi}$ denote the first and second derivatives of the function $v=v(\varphi)$ and $\lambda=t+i h$ for $r, h \in \mathbb{R}$.

Lemma 1. Suppose that $h \notin \mathbb{Z}$. Then for each $g \in L^{2}(0,2 \pi)$ there exists a unique solution $v \in H^{2}(0,2 \pi)$ of problem (3). It satisfies the estimate

$$
\|v\|_{H^{2}(0,2 \pi)}+|\lambda|\|v\|_{H^{1}(0,2 \pi)}+|\lambda|^{2}\|v\|_{L^{2}(0,2 \pi)} \leq c\|g\|_{L^{2}(0,2 \pi)},
$$

where $c$ depends only on $h$.

Proof. In this proof, $\|\cdot\|$ will stand for $\|\cdot\|_{L^{2}(0,2 \pi)}$. It can be verified that for noninteger $h$ the unique solution of $(3)$ is

$$
\begin{aligned}
v(\varphi)= & \frac{1}{2 \lambda \sinh \lambda \pi} \\
& \cdot\left(\int_{0}^{\varphi} g(\tau) \cosh \lambda(\varphi-\tau-\pi) d \tau+\int_{\varphi}^{2 \pi} g(\tau) t \cosh \lambda(\varphi-\tau+\pi) d \tau\right) .
\end{aligned}
$$

From $(3)_{1}$ we have

$$
\left\|v_{\varphi \varphi}\right\| \leq|\lambda|^{2}\|v\|+\|g\|
$$

If we multiply $(3)_{1}$ by $|\lambda|^{2} \bar{v}$ and integrate over $(0,2 \pi)$, and next utilize the boundary conditions $(3)_{2,3}$, we get

$$
|\lambda|^{2}\left\|v_{\varphi}\right\|^{2}+\lambda^{2}|\lambda|^{2}\|v\|^{2}=|\lambda|^{2} \int_{0}^{2 \pi} g \bar{v} d \tau .
$$

Hence using the Schwarz inequality we obtain

$$
|\lambda|^{2}\left\|v_{\varphi}\right\|^{2} \leq 2|\lambda|^{4}\|v\|^{2}+\|g\|^{2} .
$$

As clearly $|h| \leq|\lambda|$, using (3) 1 and (6) we get

$$
|\lambda|^{k}\|v\| \leq|h|^{k-2}\left\{\|g\|+\left\|v_{\varphi \varphi}\right\|\right\} \leq|h|^{k-2}\left\{2\|g\|+|\lambda|^{2}\|v\|\right\},
$$

where $k=0,1$. The boundary condition $(3)_{2}$ implies $\int_{0}^{2 \pi} v_{\varphi} d \varphi=0$, hence applying the Poincaré inequality and (6) we get

$$
\left\|v_{\varphi}\right\| \leq c\left\|v_{\varphi \varphi}\right\| \leq c\left\{\|g\|+|\lambda|^{2}\|v\|\right\} .
$$


Thus we have proved (4), provided

$$
|\lambda|^{2}\|v\| \leq c\|g\|
$$

for some $c=c(h)$. We shall deduce (9) in two steps. First we assume that $\lambda=t+i h$ satisfies the condition

$$
|t|>2|h| \text {. }
$$

Then comparing the real parts in (7) we get

$$
|\lambda|^{2}\left\|v_{\varphi}\right\|^{2}+\left(t^{4}-h^{4}\right)\|v\|^{2}=|\lambda|^{2} \operatorname{Re} \int_{0}^{2 \pi} g \bar{v} d \tau .
$$

The condition (10) gives us $\frac{1}{2}|\lambda|^{4} \leq t^{4}-h^{4}$, hence

$$
|\lambda|^{4}\|v\|^{2} \leq 2|\lambda|^{2} \int_{0}^{2 \pi}|g v| d \tau \leq \frac{1}{2}|\lambda|^{4}\|v\|^{2}+2\|g\|^{2} .
$$

Thus under condition (10) we deduce (9). Applying the Schwarz inequality to formula (5) we obtain the estimate

$$
|\lambda|^{4}\|v\|^{2} \leq\left|\frac{\lambda}{\sinh \lambda \pi}\right|^{2}\left(\frac{\pi}{t} \sinh 2 \pi t+\frac{\pi}{h} \sin 2 \pi h\right)\|g\|^{2},
$$

where we have used the equality

$$
\begin{aligned}
\int_{0}^{2 \pi} \int_{0}^{\varphi}|\cosh \lambda(\varphi-\tau-\pi)|^{2} d \tau d \varphi & =\int_{0}^{2 \pi} \int_{\varphi}^{2 \pi}|\cosh \lambda(\varphi-\tau+\pi)|^{2} d \tau d \varphi \\
& =\frac{\pi}{t} \sinh 2 \pi t+\frac{\pi}{h} \sin 2 \pi h .
\end{aligned}
$$

Therefore from (12) we get

$$
|\lambda|^{4}\|v\|^{2} \leq c \cdot(t+1)\|g\|^{2}
$$

where $c=c(h)$. Thus the estimate (11) for $|t|>2|h|$ and the estimate (13) for $|t| \leq 2|h|$ lead to inequality (9) with $c$ depending only on $h$.

Theorem 3. Assume that $\mu \in \mathbb{R} \backslash \mathbb{Z}$. Then for each $f \in L_{2, \mu}\left(\mathbb{R}^{2}\right)$ there exists a unique $u \in H_{\mu}^{2}\left(\mathbb{R}^{2}\right)$ such that $\Delta u=f$.

Proof. Uniqueness. Suppose that $\mu$ is noninteger and $u \in H_{\mu}^{2}\left(\mathbb{R}^{2}\right)$ satisfies $\Delta u=0$. We set

$$
\begin{aligned}
h & =1-\mu, \\
w(t, \varphi) & =u\left(e^{-t} \cos \varphi, e^{-t} \sin \varphi\right), \\
v(\lambda, \varphi) & =\frac{1}{\sqrt{2 \pi}} \int_{-\infty}^{\infty} w(t, \varphi) e^{-i \lambda t} d t .
\end{aligned}
$$


We shall show that for a.e. $\lambda \in \mathbb{R}_{h}:=\{\beta+i h: \beta \in \mathbb{R}\}$ the function $v(\lambda, \cdot)$ belongs to $H^{2}(0,2 \pi)$. Indeed,

$$
\int_{-\infty+i h}^{\infty+i h}\|v(\lambda, \cdot)\|_{H^{2}(0,2 \pi)}^{2} d \lambda \leq\|u\|_{H_{\mu}^{2}\left(\mathbb{R}^{2}\right)}^{2}<\infty
$$

because applying for $j=0,1,2$ the Parseval identity we get

$$
\int_{-\infty+i h}^{\infty+i h} \int_{0}^{2 \pi}\left|D_{\varphi}^{j} v(\lambda, \varphi)\right|^{2} d \varphi d \lambda=\int_{\mathbb{R}^{2}}\left|r^{-j} D_{\varphi}^{j} u(x)\right|^{2} r^{2 \mu-4+2 j} d x \leq\|u\|_{H_{\mu}^{2}\left(\mathbb{R}^{2}\right)}^{2} .
$$

If we write the equation $\Delta u(x)=0$ for $x \in \mathbb{R}^{2}$ in polar coordinates, substitute $t:=-\ln r$ and apply the Fourier transform with respect to the $t$ variable, we get

$$
-\lambda^{2} v(\lambda, \varphi)+D_{\varphi}^{2} v(\lambda, \varphi)=0 \quad \text { for a.e. }(\lambda, \varphi) \in \mathbb{R}_{h} \times(0,2 \pi) .
$$

Furthermore, from (15) we have

$$
w(t, 0)=w(t, 2 \pi) \quad \text { and } \quad D_{\varphi} w(t, 0)=D_{\varphi} w(t, 2 \pi) \quad \text { for a.e. } t \in \mathbb{R},
$$

thus (16) gives

$$
v(\lambda, 0)=v(\lambda, 2 \pi) \quad \text { and } \quad D_{\varphi} v(\lambda, 0)=D_{\varphi} v(\lambda, 2 \pi) \quad \text { for a.e. } \lambda \in \mathbb{R}_{h} .
$$

From (17) and (18) we see that for a.e. $\lambda \in \mathbb{R}_{h}$ the function $v(\lambda, \cdot) \in$ $H^{2}(0,2 \pi)$ satisfies the homogeneous problem (3), thus utilizing (4) we deduce that $v(\lambda, \cdot) \equiv 0$ for a.e. $\lambda \in \mathbb{R}_{h}$. Therefore (16) yields

$$
\int_{-\infty}^{\infty} w(t, \varphi) \cdot e^{-i \lambda t} d t=0 \quad \text { for a.e. }(\lambda, \varphi) \in \mathbb{R}_{h} \times(0,2 \pi)
$$

so $w(t, \varphi)=0$ for a.e. $(t, \varphi) \in \mathbb{R} \times(0,2 \pi)$, and hence $u(x)=0$ for a.e. $x \in \mathbb{R}^{2}$.

Existence. Assume that $f \in L_{2, \mu}\left(\mathbb{R}^{2}\right)$ and $\mu$ is noninteger. Then we set

$$
f_{1}(t, \varphi)=e^{-2 t} f\left(e^{-t} \cos \varphi, e^{-t} \sin \varphi\right), \quad g(\lambda, \varphi)=\int_{-\infty}^{\infty} f_{1}(t, \varphi) e^{-i \lambda t} d t .
$$

We shall show that $g(\lambda, \cdot) \in L^{2}(0,2 \pi)$ for a.e. $\lambda \in \mathbb{R}_{h}$. Indeed, applying the Parseval identity we get

$$
\int_{-\infty+i h}^{\infty+i h}\|g(\lambda, \cdot)\|_{L^{2}(0,2 \pi)}^{2} d \lambda=\|f\|_{L_{2, \mu}\left(\mathbb{R}^{2}\right)}^{2}<\infty .
$$

For $\lambda \in \mathbb{R}_{h}$ let $v(\lambda, \varphi) \in H^{2}(0,2 \pi)$ be a solution of problem (3) with r.h.s. 
$-g(\lambda, \varphi) \in L^{2}(0,2 \pi)$. Then integrating (4) with respect to $\lambda \in \mathbb{R}_{h}$ we get

$$
\begin{aligned}
\sum_{k+l \leq 2} \int_{-\infty+i h}^{\infty+i h}|\lambda|^{2 k}\left\|D_{\varphi}^{l} v(\lambda, \cdot)\right\|_{L^{2}(0,2 \pi)}^{2} d \lambda & \\
& \leq c \int_{-\infty+i h}^{\infty+i h}\|g\|_{L^{2}(0,2 \pi)}^{2} d \lambda=c\|f\|_{L_{2, \mu}\left(\mathbb{R}^{2}\right)}^{2}
\end{aligned}
$$

where $c$ depends only on $h$. We set

$$
w(t, \varphi)=\frac{1}{\sqrt{2 \pi}} \int_{-\infty+i h}^{\infty+i h} v(\lambda, \varphi) e^{i \lambda t} d \lambda
$$

and define a function $u$ on $\mathbb{R}^{2}$ by the formula

$$
u\left(e^{-t} \cos \varphi, e^{-t} \sin \varphi\right)=w(t, \varphi) \quad \text { for }(t, \varphi) \in \mathbb{R} \times(0,2 \pi) .
$$

First we shall show that $u \in H_{\mu}^{2}\left(\mathbb{R}^{2}\right)$. Indeed, applying the Parseval identity and using the boundary conditions $(3)_{2,3}$ for $v(\lambda, \cdot)$ we get

$$
\|u\|_{H_{\mu}^{2}\left(\mathbb{R}^{2}\right)}^{2} \leq \sum_{k+l \leq 2} \int_{-\infty+i h}^{\infty+i h}|\lambda|^{2 k}\left\|D_{\varphi}^{k} v(\lambda, \varphi)\right\|_{L^{2}(0,2 \pi)}^{2} d \lambda .
$$

Therefore utilizing (19) we obtain

$$
\|u\|_{H_{\mu}^{2}\left(\mathbb{R}^{2}\right)} \leq c\|f\|_{L_{2, \mu}\left(\mathbb{R}^{2}\right)},
$$

where the constant $c$ comes from (19). Finally, if we multiply

$$
\lambda^{2} v(\lambda, \varphi)-D_{\varphi}^{2} v(\lambda, \varphi)=-g(\lambda, \varphi)
$$

by $e^{i \lambda t}$ and next integrate over $\mathbb{R}_{h}$, we deduce that $\Delta u=f$, finishing the proof.

4. Estimate for the problem with parameter. In this section we prove estimates for solutions of an elliptic problem in $\mathbb{R}^{2}$ with parameter. This result is a crucial step in the proof of Theorems 1 and 2 .

REMARK 1. In the following lemma and its proof all differential operators act on the $x$ variable only. Recall that $Q_{1}, Q_{2}$ and $s=s(q)$ were defined in Section 2 .

Lemma 2. Assume that $\mu \in(-1,0), n=-1,0,1,2, \ldots, i=1,2$ and $v$ is a smooth function on $\mathbb{R}^{2} \times Q_{i}$ with bounded support. Assume that $v(\cdot, q) \in$ 
$\mathcal{D}\left(\dot{\mathbb{R}}^{2}\right)$ for each $q \in Q_{i}$. Then there exists a constant $c=c(\mu, n)$ such that

$$
\begin{aligned}
\int_{Q_{i} \mathbb{R}^{2}}\left[\left|D^{2} v(x, q)\right|^{2}+|s||\nabla v(x, q)|^{2}\right. & \left.+|s|^{2}|v(x, q)|^{2}\right]|x|^{2 \mu-2 n} d x d q \\
& \leq c \int_{Q_{i} \mathbb{R}^{2}}|g(x, q)|^{2}|x|^{2 \mu-2 n} d x d q
\end{aligned}
$$

where

$$
g(x, q)=-\Delta v(x, q)+s v(x, q) .
$$

Proof. In the case of $n=-1$ the proof of (20) can be found in [Ku07] (see Lemma 4 and Remark 4). Therefore we only have to show (20) for $n$ a nonnegative integer. So suppose that $v$ satisfies the assumptions. If we multiply $(21)$ by $\bar{\varphi} \in H^{1}\left(\mathbb{R}^{2}\right)$, integrate with respect to $x \in \mathbb{R}^{2}$ and integrate by parts, then for all $\varphi \in H^{1}\left(\mathbb{R}^{2}\right)$ we obtain

$$
\int_{\mathbb{R}^{2}}[\nabla v(x, q) \cdot \nabla \bar{\varphi}(x)+s v(x, q) \bar{\varphi}(x)] d x=\int_{\mathbb{R}^{2}} g(x, q) \bar{\varphi}(x) d x .
$$

Since $v(\cdot, q) \in \mathcal{D}\left(\dot{\mathbb{R}}^{2}\right)$ for each $q \in Q_{i}$, we see that $\varphi:=(1+i \operatorname{sgn} b) v|x|^{2 \mu-2 n} \in$ $H^{1}\left(\mathbb{R}^{2}\right)$, where $b:=\operatorname{Im} s$. Putting $\varphi$ in (22) yields

$$
\begin{aligned}
& \int_{\mathbb{R}^{2}}\left\{|\nabla v|^{2}|x|^{2 \mu-2 n}(1-i \operatorname{sgn} b)+\nabla v \cdot \nabla\left(|x|^{2 \mu-2 n}\right) \bar{v}(1-i \operatorname{sgn} b)\right\} d x \\
& \quad+(1-i \operatorname{sgn} b) s \int_{\mathbb{R}^{2}}|v|^{2}|x|^{2 \mu-2 n} d x=(1-i \operatorname{sgn} b) \int_{\mathbb{R}^{2}} g \bar{v}|x|^{2 \mu-2 n} d x .
\end{aligned}
$$

Comparing the real parts we get

$$
\begin{aligned}
& \int_{\mathbb{R}^{2}}\left(|\nabla v|^{2}+|s||v|^{2}\right)|x|^{2 \mu-2 n} d x \\
& \quad \leq 4|\mu-n| \int_{\mathbb{R}^{2}}|\nabla v||v||x|^{2 \mu-2 n-1} d x+2 \int_{\mathbb{R}^{2}}|g||v||x|^{2 \mu-2 n} d x .
\end{aligned}
$$

Multiplying both sides by $|s|$ and next applying twice the Young inequality we get

$$
\begin{aligned}
|s| \int_{\mathbb{R}^{2}}\left(|\nabla v|^{2}+|s||v|^{2}\right)|x|^{2 \mu-2 n} d x & \\
\leq & \frac{1}{2}|s| \int_{\mathbb{R}^{2}}|\nabla v|^{2}|x|^{2 \mu-2 n} d x+2^{3}(n+1)^{2}|s| \int_{\mathbb{R}^{2}}|v|^{2}|x|^{2 \mu-2 n-2} d x \\
& +2 \int_{\mathbb{R}^{2}}|g|^{2}|x|^{2 \mu-2 n} d x+\frac{1}{2}|s|^{2} \int_{\mathbb{R}^{2}}|v|^{2}|x|^{2 \mu-2 n} d x
\end{aligned}
$$


hence

$$
\begin{aligned}
& |s| \int_{\mathbb{R}^{2}}\left(|\nabla v|^{2}+|s||v|^{2}\right)|x|^{2 \mu-2 n} d x \\
& \quad \leq 2^{4}(n+1)^{2}|s| \int_{\mathbb{R}^{2}}|v|^{2}|x|^{2 \mu-2 n-2} d x+4 \int_{\mathbb{R}^{2}}|g|^{2}|x|^{2 \mu-2 n} d x .
\end{aligned}
$$

We set

$$
\begin{aligned}
& A_{1}:=\left\{(x, q) \in \mathbb{R}^{2} \times Q_{i} ;|s||x|^{2}<a_{1}\right\}, \\
& A_{2}:=\left\{(x, q) \in \mathbb{R}^{2} \times Q_{i} ; a_{1} \leq|s||x|^{2}<a_{2}\right\}, \\
& A_{3}:=\left\{(x, q) \in \mathbb{R}^{2} \times Q_{i} ; a_{2} \leq|s||x|^{2}\right\},
\end{aligned}
$$

where the numbers $0<a_{1}<a_{2}$ will be chosen later. For $q \in Q_{i}$ we write

$$
\begin{aligned}
& d_{1}(q):=\left\{x \in \mathbb{R}^{2} ;|s||x|^{2}<a_{1}\right\}, \\
& d_{2}(q):=\left\{x \in \mathbb{R}^{2} ; a_{1} \leq|s||x|^{2}<a_{2}\right\}, \\
& d_{3}(q):=\left\{x \in \mathbb{R}^{2} ; a_{2} \leq|s||x|^{2}\right\} .
\end{aligned}
$$

For $\lambda>0$ and $q \in Q_{i}$ we define

$$
\Omega^{\lambda}:=\left\{(x, q) \in \mathbb{R}^{2} \times Q_{i} ; \lambda|s||x|^{2}<1\right\}, \quad \omega^{\lambda}(q):=\left\{x \in \mathbb{R}^{2} ; \lambda|s||x|^{2}<1\right\} .
$$

Clearly,

$$
A_{2} \subseteq \Omega^{\lambda} \quad \text { for } \lambda \in\left(0, a_{2}^{-1}\right)
$$

On $A_{1}$ we have the estimate $|s| \leq a_{1}|x|^{-2}$, thus

$$
\begin{aligned}
\int_{A_{1}}|s||v|^{2}|x|^{2 \mu-2 n-2} d x d q & \leq a_{1} \int_{A_{1}}|v|^{2}|x|^{2 \mu-2 n-4} d x d q \\
& \leq a_{1} \int_{Q_{i}}\|v(\cdot, q)\|_{H_{\mu-n}^{2}\left(\mathbb{R}^{2}\right)}^{2} d q .
\end{aligned}
$$

We deduce from the assumptions that for each $q \in Q_{i}$ the function $v(\cdot, q)$ is in $H_{\mu-n}^{2}\left(\mathbb{R}^{2}\right)$ and satisfies the equation $-\Delta v=g-s v$. The right hand side is in $L_{2, \mu-n}\left(\mathbb{R}^{2}\right)$, thus from Theorem 3 we get a constant $c_{0}=c_{0}(\mu-n)$ such that

$$
\|v(\cdot, q)\|_{H_{\mu-n}^{2}\left(\mathbb{R}^{2}\right)}^{2} \leq c_{0}\|g(\cdot, q)\|_{L_{2, \mu-n}\left(\mathbb{R}^{2}\right)}^{2}+c_{0}|s|^{2}\|v(\cdot, q)\|_{L_{2, \mu-n}\left(\mathbb{R}^{2}\right)}^{2}
$$

for each $q \in Q_{i}$. Hence, from (25) we get

$$
\begin{aligned}
& \int_{A_{1}}|s||v|^{2}|x|^{2 \mu-2 n-2} d x d q \\
& \quad \leq a_{1} c_{0} \int_{Q_{i}} \int_{\mathbb{R}^{2}}|g|^{2}|x|^{2 \mu-2 n} d x d q+a_{1} c_{0} \int_{Q_{i}}|s|^{2} \int_{\mathbb{R}^{2}}|v|^{2}|x|^{2 \mu-2 n} d x d q .
\end{aligned}
$$


On $A_{3}$ we have $|x|^{-2} \leq a_{2}^{-1}|s|$, thus

$$
\int_{A_{3}}|s||v|^{2}|x|^{2 \mu-2 n-2} d x d q \leq a_{2}^{-1} \int_{A_{3}}|s|^{2}|v|^{2}|x|^{2 \mu-2 n} d x d q .
$$

On $A_{2}$ we have $|x|^{-2} \leq a_{1}^{-1}|s|$, thus $|x|^{2 \mu-2 n-2} \leq a_{1}^{\mu-n-1}|s|^{1+n-\mu}$ and

$$
\int_{A_{2}}|s||v|^{2}|x|^{2 \mu-2 n-2} d x d q \leq a_{1}^{\mu-n-1} \int_{A_{2}}|s|^{2+n-\mu}|v|^{2} d x d q
$$

If we integrate $(23)$ with respect to $q \in Q_{i}$, then according to (27)-(29) we get

$$
\begin{aligned}
& \int_{Q_{i}}|s| \int_{\mathbb{R}^{2}}\left(|\nabla v|^{2}+|s||v|^{2}\right)|x|^{2 \mu-2 n} d x d q \\
& \leq 2^{4}(n+1)^{2}\left(a_{2}^{-1}+a_{1} c_{0}\right) \int_{Q_{i}}|s|^{2} \int_{\mathbb{R}^{2}}|v|^{2}|x|^{2 \mu-2 n} d x d q \\
& \quad+\left(4+a_{1} c_{0}\right) \int_{Q_{i}} \int_{\mathbb{R}^{2}}|g|^{2}|x|^{2 \mu-2 n} d x d q+2^{4}(n+1)^{2} a_{1}^{\mu-n-1} \int_{A_{2}}|s|^{2+n-\mu}|v|^{2} d x d q .
\end{aligned}
$$

We put $a_{1}:=2^{-6} c_{0}^{-1}(n+1)^{-2}$ and $a_{2}:=2^{6}(n+1)^{2}$ to obtain

$$
\begin{aligned}
& \int_{Q_{i}}|s| \int_{\mathbb{R}^{2}}\left(|\nabla v|^{2}+|s||v|^{2}\right)|x|^{2 \mu-2 n} d x d q \\
& \quad \leq 10 \int_{Q_{i}} \int_{\mathbb{R}^{2}}|g|^{2}|x|^{2 \mu-2 n} d x d q+c(\mu, n) \int_{A_{2}}|s|^{2+n-\mu}|v|^{2} d x d q .
\end{aligned}
$$

Let $\chi=\chi(t)$ be a smooth cut-off function such that $\chi(t)=1$ if $|t| \leq 1$ and $\chi(t)=0$ if $|t| \geq 2,0 \leq \chi(t) \leq 1,\left|\chi^{\prime}(t)\right| \leq 4$. We write

$$
\chi_{\lambda}(x, q):=\chi\left(\lambda|s||x|^{2}\right) .
$$

Then $\chi_{\lambda}(x, q)=1$ if $|s||x|^{2}<\lambda^{-1}$ and $\chi_{\lambda}(x, q)=0$ if $|s||x|^{2}>2 \lambda^{-1}$. Clearly,

$$
\left|\nabla \chi_{\lambda}(x, q)\right| \leq 8 \lambda|s||x|
$$

We put $\varphi:=(1+i \operatorname{sgn} b) v \chi_{\lambda}^{2} \in H^{1}\left(\mathbb{R}^{2}\right)$ in $(22)$ to get

$$
\begin{aligned}
(1-i \operatorname{sgn} b) \int_{\mathbb{R}^{2}}\left(|\nabla v|^{2} \chi_{\lambda}^{2}+\right. & \left.2 \bar{v} \chi_{\lambda} \nabla v \cdot \nabla \chi_{\lambda}+s|v|^{2} \chi_{\lambda}^{2}\right) d x \\
& =(1-i \operatorname{sgn} b) \int_{\mathbb{R}^{2}} g \bar{v} \chi_{\lambda}^{2} d x \quad \text { for each } q \in Q_{i}
\end{aligned}
$$


Comparing the real parts we get

$$
\int_{\mathbb{R}^{2}}\left(|\nabla v|^{2}+|s||v|^{2}\right) \chi_{\lambda}^{2} d x \leq 2 \sqrt{2} \int_{\mathbb{R}^{2}}|\nabla v|\left|\nabla \chi_{\lambda}\right||v| \chi_{\lambda} d x+\sqrt{2} \int_{\mathbb{R}^{2}}|g||v| \chi_{\lambda}^{2} d x .
$$

Applying twice the Young inequality, we get

$$
\begin{gathered}
\int_{\mathbb{R}^{2}}\left(|\nabla v|^{2}+|s||v|^{2}\right) \chi_{\lambda}^{2} d x \leq \frac{1}{2} \int_{\mathbb{R}^{2}}|\nabla v|^{2} \chi_{\lambda}^{2} d x+4 \int_{\mathbb{R}^{2}}\left|\nabla \chi_{\lambda}\right|^{2}|v|^{2} d x \\
+2^{-1} \varepsilon|s|^{n+1-\mu} \int_{\mathbb{R}^{2}}|v|^{2} \chi_{\lambda}^{2}|x|^{2 n-2 \mu} d x+2 \varepsilon^{-1}|s|^{\mu-1-n} \int_{\mathbb{R}^{2}}|g|^{2} \chi_{\lambda}^{2}|x|^{2 \mu-2 n} d x .
\end{gathered}
$$

On the support of $\chi_{\lambda}$ we have $|s|^{n-\mu}|x|^{2 n-2 \mu} \leq 2^{n-\mu} \lambda^{\mu-n}$, hence

$$
2^{-1} \varepsilon|s|^{n+1-\mu} \int_{\mathbb{R}^{2}}|v|^{2} \chi_{\lambda}^{2}|x|^{2 n-2 \mu} d x \leq \frac{\varepsilon}{2}\left(\frac{2}{\lambda}\right)^{n-\mu} \int_{\mathbb{R}^{2}}|s||v|^{2} \chi_{\lambda}^{2} d x .
$$

We set $\varepsilon:=(\lambda / 2)^{n-\mu}$. Then (33) and (34) give the estimate

$$
\begin{aligned}
& \int_{\mathbb{R}^{2}}\left(|\nabla v|^{2}+|s||v|^{2}\right) \chi_{\lambda}^{2} d x \\
& \quad \leq 8 \int_{\mathbb{R}^{2}}\left|\nabla \chi_{\lambda}\right|^{2}|v|^{2} d x+4\left(\frac{2}{\lambda}\right)^{n-\mu}|s|^{\mu-1-n} \int_{\mathbb{R}^{2}}|g|^{2} \chi_{\lambda}^{2}|x|^{2 \mu-2 n} d x .
\end{aligned}
$$

We multiply both sides by $|s|^{n+1-\mu}$ and integrate with respect to $q$ to get

$$
\begin{aligned}
& \int_{Q_{i}}|s|^{n+1-\mu} \int_{\mathbb{R}^{2}}\left(|\nabla v|^{2}+|s||v|^{2}\right) \chi_{\lambda}^{2} d x d q \\
& \quad \leq 8 \int_{Q_{i}}|s|^{n+1-\mu} \int_{\mathbb{R}^{2}}\left|\nabla \chi_{\lambda}\right|^{2}|v|^{2} d x d q+4\left(\frac{2}{\lambda}\right)^{n-\mu} \int_{Q_{i}} \int_{\mathbb{R}^{2}}|g|^{2} \chi_{\lambda}^{2}|x|^{2 \mu-2 n} d x d q .
\end{aligned}
$$

According to the definition of $\chi_{\lambda}$ and the property (32) we have $\left|\nabla \chi_{\lambda}\right|^{2} \leq$ $2^{7} \lambda|s|$, thus

$$
\begin{aligned}
\int_{Q_{i}}|s|^{n+1-\mu} \int_{\mathbb{R}^{2}}\left(|\nabla v|^{2}+|s||v|^{2}\right) & \chi_{\lambda}^{2} d x d q \\
\leq & 2^{10} \lambda \int_{Q_{i}}|s|^{n+2-\mu} \int_{\omega^{1 / 2}(q) \backslash \omega^{\lambda}(q)}|v|^{2} d x d q \\
& +4\left(\frac{2}{\lambda}\right)^{n-\mu} \int_{Q_{i} \mathbb{R}^{2}}|g|^{2} \chi_{\lambda}^{2}|x|^{2 \mu-2 n} d x d q .
\end{aligned}
$$


Hence multiplying both sides by $(\lambda / 2)^{n-\mu}$ we obtain

$$
\begin{gathered}
\left(\frac{\lambda}{2}\right)^{n-\mu} \int_{Q_{i}}|s|^{n+1-\mu} \int_{\mathbb{R}^{2}}\left(|\nabla v|^{2}+|s||v|^{2}\right) \chi_{\lambda}^{2} d x d q \\
\leq 2^{10+n-\mu} \lambda\left(\frac{\lambda}{2^{2}}\right)^{n-\mu} \int_{Q_{i}}|s|^{2+n-\mu} \int_{\omega^{1 / 2}(q) \backslash \omega^{\lambda}(q)}|v|^{2} d x d q \\
+4 \int_{Q_{i} \mathbb{R}^{2}}|g|^{2} \chi_{\lambda}^{2}|x|^{2 \mu-2 n} d x d q .
\end{gathered}
$$

We assume that $\lambda<2^{-12-n}$. Thus we get

$$
\begin{aligned}
\left(\frac{\lambda}{2}\right)^{n-\mu} \int_{Q_{i}}|s|^{n+2-\mu} & \int_{\omega^{\lambda}(q)}|v|^{2} d x d q \\
\leq & \frac{1}{2}\left(\frac{\lambda}{2^{2}}\right)^{n-\mu} \int_{Q_{i}}|s|^{n+2-\mu} \int_{\omega^{\lambda / 2}(q)}|v|^{2} d x d q+K,
\end{aligned}
$$

where $K:=4 \int_{Q} \int_{\mathbb{R}^{2}}|g|^{2}|x|^{2 \mu-2 n} d x d q$. We denote by $f(\lambda)$ the expression on the 1.h.s. of (35). Thus the above estimate has the form

$$
f(\lambda) \leq \frac{1}{2} f(\lambda / 2)+K
$$

and holds for each $\lambda$ in $\left(0,2^{-12-n}\right)$. Iterating this estimate we get

$$
f(\lambda) \leq \frac{1}{2^{k}} f\left(\lambda / 2^{k}\right)+\left(2-2^{1-k}\right) K
$$

for each $\lambda \in\left(0,2^{-12-n}\right)$ and $k=1,2, \ldots$ On the set $\omega^{\lambda / 2^{k}}(q)$ we have $|s|^{n-\mu} \leq\left(2^{k} / \lambda\right)^{n-\mu}|x|^{2 \mu-2 n}$, hence

$$
f\left(\lambda / 2^{k}\right) \leq \int_{Q_{i}}|s|^{2} \int_{\mathbb{R}^{2}}|v|^{2}|x|^{2 \mu-2 n} d x d q .
$$

We fix $\lambda_{0} \in\left(0,2^{-12-n}\right)$. Then from (24) we deduce that $A_{2} \subseteq \Omega^{\lambda_{0}}$. Applying the estimate (30), the definition of $f\left(\lambda_{0}\right)$ and the estimates (37) and (38) we obtain

$$
\begin{aligned}
& \int_{Q_{i}}|s| \int_{\mathbb{R}^{2}}\left(|\nabla v|^{2}+|s||v|^{2}\right)|x|^{2 \mu-2 n} d x d q \\
& \quad \leq 3 K+c(\mu, n) \int_{A_{2}}|s|^{n+2-\mu}|v|^{2} d x d q \leq 3 K+c(\mu, n) \int_{\Omega^{\lambda_{0}}}|s|^{n+2-\mu}|v|^{2} d x d q
\end{aligned}
$$




$$
\begin{aligned}
& =3 K+c(\mu, n)\left(\frac{2}{\lambda_{0}}\right)^{n-\mu} f\left(\lambda_{0}\right) \leq c K+c(\mu, n)\left(\frac{2}{\lambda_{0}}\right)^{n-\mu} \frac{1}{2^{k}} f\left(\lambda_{0} / 2^{k}\right) \\
& \leq K+c(\mu, n)\left(\frac{2}{\lambda_{0}}\right)^{n-\mu} \frac{1}{2^{k}} \int_{Q_{i}}|s|^{2} \int_{\mathbb{R}^{2}}|v|^{2}|x|^{2 \mu-2 n} d x d q,
\end{aligned}
$$

where $c=c\left(c_{0}, \lambda_{0}, \mu, n\right)$. Now letting $k \rightarrow \infty$, we get

$$
\int_{Q_{i}}|s| \int_{\mathbb{R}^{2}}\left(|\nabla v|^{2}+|s||v|^{2}\right)|x|^{2 \mu-2 n} d x d q \leq c K .
$$

Finally, applying (26) we get the estimate (20).

\section{Proof of the main results}

Proof of Theorem 1. The space $\mathcal{D}\left(\dot{\mathbb{R}}^{3}\right)$ is dense in $H_{\mu}^{2}\left(\mathbb{R}^{3}\right)$, therefore it is enough to show $(1)$ for $u \in \mathcal{D}\left(\dot{\mathbb{R}}^{3}\right)$ with a constant $c$ independent of $u$. For $u \in \mathcal{D}\left(\dot{\mathbb{R}}^{3}\right), x^{\prime} \in \mathbb{R}^{2}$ and $q \in \mathbb{R}$ we denote by $\widehat{u}\left(x^{\prime}, q\right)$ the partial Fourier transform with respect to $z$, i.e.

$$
\widehat{u}\left(x^{\prime}, q\right):=\frac{1}{\sqrt{2 \pi}} \int_{-\infty}^{\infty} u\left(x^{\prime}, z\right) e^{-i z q} d z .
$$

Clearly, $\widehat{u}$ satisfies the assumptions of Lemma 2 for $Q_{1}$. Thus we get a constant $c=c(\mu, n)$ such that

$$
\begin{aligned}
\iint_{\mathbb{R}}\left(\left|D_{x^{\prime}}^{2} \widehat{u}\right|^{2}+|q|^{2}\left|D_{x^{\prime}} \widehat{u}\right|^{2}+|q|^{4}|\widehat{u}|^{2}\right)\left|x^{\prime}\right|^{2 \mu-2 n} d x d q & \\
& \leq c \iint_{\mathbb{R} \mathbb{R}^{2}}|\widehat{f}|^{2}\left|x^{\prime}\right|^{2 \mu-2 n} d x d q
\end{aligned}
$$

where

$$
\widehat{f}\left(x^{\prime}, q\right)=-\Delta_{x^{\prime}} \widehat{u}\left(x^{\prime}, q\right)+s \widehat{u}\left(x^{\prime}, q\right) \text { and } s=q^{2} .
$$

Applying the Hardy inequality ([Ha34, Theorem 330]) we get

$$
\begin{gathered}
\int_{\mathbb{R}^{2}}\left(|\widehat{u}|^{2}\left|x^{\prime}\right|^{-4}+\left|D_{x^{\prime}} \widehat{u}\right|^{2}\left|x^{\prime}\right|^{-2}\right)\left|x^{\prime}\right|^{2 \mu-2 n} d x \leq c \int_{\mathbb{R}^{2}}\left|D_{x^{\prime}}^{2} \widehat{u}\right|^{2}\left|x^{\prime}\right|^{2 \mu-2 n} d x, \\
\int_{\mathbb{R}^{2}}|\widehat{u}|^{2}\left|x^{\prime}\right|^{2 \mu-2 n-2} d x \leq c \int_{\mathbb{R}^{2}}\left|D_{x^{\prime}} \widehat{u}\right|^{2}\left|x^{\prime}\right|^{2 \mu-2 n} d x
\end{gathered}
$$


for all $q \in Q$, where $c=c(\mu, n)$. Thus from (39)-(41) we obtain $\iint\left(\left|D_{x^{\prime}}^{2} \widehat{u}\right|^{2}+|q|^{2}\left|D_{x^{\prime}} \widehat{u}\right|^{2}+|q|^{4}|\widehat{u}|^{2}\right)\left|x^{\prime}\right|^{2 \mu-2 n} d x d q$

$\mathbb{R} \mathbb{R}^{2}$

$$
\begin{array}{r}
+\int_{\mathbb{R} \mathbb{R}^{2}}\left(|\widehat{u}|^{2}\left|x^{\prime}\right|^{-4}+\left|D_{x^{\prime}} \widehat{u}\right|^{2}\left|x^{\prime}\right|^{-2}+|q|^{2}|\widehat{u}|^{2}\left|x^{\prime}\right|^{-2}\right)\left|x^{\prime}\right|^{2 \mu-2 n} d x d q \\
\quad \leq c \iint_{\mathbb{R} \mathbb{R}^{2}}|\widehat{f}|^{2}\left|x^{\prime}\right|^{2 \mu-2 n} d x d q
\end{array}
$$

for some $c=c(\mu, n)$. Applying the Parseval identity to both sides of the above inequality we deduce the estimate (1) for all $u \in \mathcal{D}\left(\dot{\mathbb{R}}^{3}\right)$.

Proof of Theorem 2. Because $\mathcal{D}\left(\dot{\mathbb{R}}^{3} \times \mathbb{R}_{+}\right)$is dense in $H_{\mu}^{2,1}\left(\dot{\mathbb{R}}^{3} \times \mathbb{R}_{+}\right)$, it is enough to show $(2)$ for $u \in \mathcal{D}\left(\dot{\mathbb{R}}^{3} \times \mathbb{R}_{+}\right)$. For $q=\left(q_{1}, q_{2}\right) \in \mathbb{R}^{2}$ we write

$$
v\left(x^{\prime}, q\right):=\frac{1}{2 \pi} \int_{-\infty}^{\infty} \int_{-\infty}^{\infty} u\left(x^{\prime}, z, t\right) e^{-i\left(z q_{1}+t q_{2}\right)} d z d t
$$

where we set $u=0$ for $t<0$. The function $v\left(x^{\prime}, q\right)$ satisfies the assumptions of Lemma 2 for $Q_{2}$. Hence

$$
\begin{array}{r}
\int_{Q} \int_{\mathbb{R}^{2}}\left[\left|D^{2} v\left(x^{\prime}, q\right)\right|^{2}+|s|\left|\nabla v\left(x^{\prime}, q\right)\right|^{2}+|s|^{2}\left|v\left(x^{\prime}, q\right)\right|^{2}\right]\left|x^{\prime}\right|^{2 \mu-2 n} d x^{\prime} d q \\
\quad \leq c \iint_{Q \mathbb{R}^{2}}\left|g\left(x^{\prime}, q\right)\right|^{2}\left|x^{\prime}\right|^{2 \mu-2 n} d x^{\prime} d q
\end{array}
$$

where

$$
g\left(x^{\prime}, q\right):=-\Delta_{x^{\prime}} v\left(x^{\prime}, q\right)+s v\left(x^{\prime}, q\right) \text { and } s=q_{1}^{2}+i q_{2} .
$$

Utilizing the Hardy inequality as in (40)-(41) and next applying the Parseval identity we deduce (2).

\section{References}

[Ha34] G. H. Hardy, J. E. Littlewood and G. Pólya, Inequalities, Cambridge Univ. Press, 1934.

[Ko67] V. A. Kondrat'ev, Boundary value problems for elliptic equations in domains with conical or angular points, Trudy Moskov. Mat. Obshch. 16 (1967), 209-292 (in Russian).

[Ko97] V. A. Kozlov, V. G. Maz'ya and J. Rossmann, Elliptic Boundary Value Problems in Domains with Point Singularities, Amer. Math. Soc., Providence, RI, 1997.

[Ku07] A. Kubica and W. M. Zajączkowski, Parabolic system in weighted Sobolev space, Appl. Math. (Warsaw) 34 (2007), 169-191.

[Mc72] B. Muckenhoupt, Weighted norm inequalities for the Hardy maximal function, Trans. Amer. Math. Soc. 165 (1972), 207-226. 
[Na94] S. A. Nazarov and B. A. Plamenevsky, Elliptic Problems in Domains with Piecewise Smooth Boundaries, de Gruyter, Berlin, 1994.

[Za04] W. M. Zajączkowski, Global special regular solutions to the Navier-Stokes equations in a cylindrical domain under boundary slip conditions, Gakuto Internat. Ser. Math. Sci. Appl. 21, Gakkōtosho, Tokyo, 2004.

Institute of Mathematics and Cryptology

Military University of Technology

Kaliskiego 2

00-908 Warszawa, Poland

and

Faculty of Mathematics and Information Science

Warsaw University of Technology

Pl. Politechniki 1

00-661 Warszawa, Poland

E-mail: a.kubica@mini.pw.edu.pl
Institute of Mathematics and Cryptology

Military University of Technology

Kaliskiego 2

00-908 Warszawa, Poland

and

Institute of Mathematics Polish Academy of Sciences

Sniadeckich 8

00-956 Warszawa, Poland

E-mail: wz@impan.gov.pl

Received on 26.2.2007;

revised version on 10.10.2007 